\title{
LAS PAUSAS SILENTES Y ORALIZADAS EN ORACIONES SUBORDINADAS SUSTANTIVAS OBJETIVAS PRONUNCIADAS POR HABLANTES DE IQUIQUE Y PUNTA ARENAS
}

Silent and oralic pauses in subordinate, objetive and subjective sentences, pronounced by speakers from Iquique and Punta Arenas

\author{
Sonia Hernández Rodríguez* \\ Jaime Soto-Barba**
}

\section{Resumen}

En el marco de un estudio mayor, cuyo principal objetivo es postular la existencia de diferencias entonativas entre zonas lingüísticas de Chile en la emisión de oraciones subordinadas sustantivas objetivas, en este trabajo se expone una caracterización de tres tipos de pausas (silencios, alargamientos y vacilaciones) halladas al interior de las estructuras oracionales indicadas anteriormente, emitidas por hablantes profesionales provenientes de Iquique y de Punta Arenas. El propósito principal del estudio consistió en determinar si se manifestaban diferencias en los tipos de pausas observadas en relación con la complejidad oracional analizada, el género de los informantes y su procedencia geográfica. Para ello se analizaron 100 enunciados extraídos de entrevistas realizadas a 37 profesionales (hombres y mujeres) nacidos en ambas ciudades. Los resultados indicaron que hubo diferencias en las pausas observadas, en relación con los tipos de oraciones analizadas, la procedencia geográfica y el género de los informantes.

Palabras clave: Pausas (silencios, alargamientos, vacilaciones), habla espontánea, Iquique vs Punta Arenas, hombres vs mujeres.

\section{Abstract}

As part of a larger study, whose main objective is to postulate the existence of intonation differences between linguistic regions of Chile in the issuance of subordinate clauses substantive objective, this work exposes a characterization of three types of pauses (silence, elongations and vacillations) found inside the sentence structures listed above, issued by professional speakers from Iquique and Punta Arenas. The main purpose of the study was to determine the existence of differences in the types of pauses observed in relation to the sentence complexity analyzed, the gender of the informants and their geographical origin. For this, a total of 100 statements taken from interviews of 37 professionals (men and women) born in both cities were analyzed. The results indicated that there were differences in the observed pauses, in relation to the types of sentences analyzed, the geographic origin and gender of the informants.

Key words: Pauses (silences, elongations, vacillations), spontaneous speech, Iquique vs Punta Arenas, men $v s$ women. 


\section{INTRODUCCIÓN}

Analizar el nivel suprasegmental del discurso oral constituye una "aventura peligrosa" (Hidalgo, 1997) cuando el registro del que se parte considera este nivel como el sistema cohesivo en el que es posible comprender y explicar la verdadera estructura sintáctico-semántica y pragmática de la lengua. Por esta razón, prestar atención al estudio prosódico de manifestaciones orales auténticas y reales sin prescindir de aquellos elementos que dificultan su análisis, así como las pausas, el orden de las palabras, la atenuación, la entonación, la vacilación, los falsos comienzos, etc., y que son tan relevantes en su contexto de uso, permite reconocer el valor prioritario de los mismos a la hora de segmentar, organizar, estructurar y comprender el discurso oral. Según esto, los recursos prosódicos son los encargados de dotar de relevancia comunicativa a las secuencias de habla. De allí que su análisis constituya una prioridad en la búsqueda de criterios sólidos que faciliten la descripción y posible sistematización de las funciones pragmático-discursivas presentes en todo acto de lenguaje.

La pausa es considerada el criterio prosódico demarcativo más evidente. Aunque podría relacionarse de manera directa con una de las tres condiciones biológicamente determinadas que corresponde a la fase de exhalación del proceso respiratorio, y a la forma en la que esta conduce a una caída subglótica de la presión del aire hacia el final de los enunciados y que tiene que ver con los límites entre grupos entonativos (Gussenhoven, 2002), la pausa constituye un parámetro prosódico que, además de intervenir en la estructuración del habla como elemento delimitador de unidades discursivas, juega un papel fundamental en la semantización de la información (Blondet, 2006) como reflejo del proceso cognitivo de codificación del lenguaje. En líneas generales, al interior del intercambio comunicativo la pausa representa un breve intervalo temporal empleado por el hablante como la estrategia mental que le permite diseñar y planificar verbalmente su discurso, reflejo de las fases de desarrollo del proceso de codificación lingüística.

La pausa se puede manifestar en el límite de los constituyentes mayores y ante palabras de alto contenido léxico o constituyentes menores (Cruttenden, 1990) con una función netamente delimitadora; sin embargo, además de delimitar, puede establecer jerarquías de unidades sintácticas según la duración que presente al interior de la cadena hablada (Cruttenden, 1990; Cazanove citado por Hidalgo, 1997), así como también puede ser indicadora de información lingüística y extralingüística de diversa índole (Blondet, 2006).

Sin embargo, la pausa silenciosa puede verse reemplazada por un alargamiento de la sílaba final de un contorno con el objetivo de determinar el cierre de un grupo entonativo. Asimismo, puede manifestarse mediante una vacilación expresada por medio de la articulación de una vocal neutra, de una nasal (bilabial o alveolar) o una continuación glotal (Crystal, 1971) de duración diversa, mediante esto el hablante expresa incertidumbre o busca prolongar su intervención evitando una interrupción repentina del interlocutor. Este tipo de pausas se conocen como pausas llenas u oralizadas. 
El análisis de este tipo de pausas ha sido abordado desde diversos ámbitos. En efecto, tras el objetivo de mejorar los sistemas de reconocimiento del habla, el campo de las tecnologías ha observado la duración de las pausas como un posible parámetro diferenciador de variabilidad dialectal y, de igual forma, en el ámbito de la adquisición de segundas lenguas, el estudio de las pausas ha buscado indagar la relación de dependencia de las mismas con conductas presentes en el uso de la lengua materna, por parte del hablante, o como posible indicador de fluidez en la producción de la segunda lengua (Machuca et al., 2015). Asimismo, las pausas llenas u oralizadas han constituido parámetros consistentes para caracterizar las alteraciones lingüísticas de pacientes con Alzheimer como manifestación directa de la dificultad léxica y cognitiva que sufren en el proceso de comprensión de la situación comunicativa y en el mecanismo de respuesta ante la misma en la producción oral (Valdivieso et al., 2003; Barkat y Gayraud, 2009). Igualmente, la pausa ha sido analizada desde el ámbito judicial como un posible fenómeno para identificar a los hablantes por la constancia y preferencia de su uso al interior del discurso mediante la observación de un conjunto de variables fonéticoacústicas referentes a la calidad vocálica de la voz en los segmentos correspondientes a las pausas llenas (Cicres, 2007).

Aunque se podrían mencionar los diversos acercamientos lingüísticos que se han realizado en torno a la búsqueda de una explicación del papel comunicativo que juegan las pausas silenciosas y las pausas oralizadas al interior de los actos de habla y su relevancia como elementos indicadores de transición al interior de la conversación, es clara la ausencia de trabajos que busquen proporcionar datos acerca de estos dos tipos de pausas en la lengua española, que enmarquen su objeto de estudio en un tipo específico de enunciado y en una situación comunicativa espontánea dada.

En Chile, la pausa ha sido escasamente abordada como un objeto central de estudio y cuando lo ha sido, ha constituido, en la mayoría de los trabajos revisados, un mecanismo empleado para delimitar unidades de análisis al interior del discurso. TapiaLadino y Valdivieso (2000), mediante una aproximación general a aspectos del procedimiento de análisis de la producción del habla, presentan una revisión de diferentes trabajos teóricos y prácticos de los aspectos temporales del habla. Este objetivo los lleva a concluir que, a pesar de que las pausas se asocian al trabajo cognitivo, como reflejo del funcionamiento del lenguaje en la mente, cumplen a su vez otras funciones comunicativas al interior del discurso que es necesario considerar a la hora de analizar un fenómeno prosódico como este. Sin embargo, su estudio se limita a la exposición de diversas posturas teóricas, pero no plantea una propuesta que permita establecer criterios comunes para clasificar "las pausas según su función: pausas de búsqueda y planificación, de aquellas pausas con función fática" (Tapia-Ladino y Valdivieso, 2000, p. 126), ni alude a la aplicación de este objetivo en algún corpus de habla específico. Por su parte, Ortiz-Lira (1999) menciona la pausa como indicador de los límites de las frases entonacionales. Al exponer las cuatro capas paralelas del sistema de notación prosódica ToBI, aborda la pausa llena y la pausa vacía en dos capas 
independientes. En la capa ortográfica, reconoce que la flexibilidad de ToBI le permite a cada transcriptor decidir en torno a la información que desea incluir en su estudio. De allí que las pausas llenas no necesariamente son abordadas como fenómenos relevantes en el análisis. Mientras que en la capa de las junturas, las pausas vacías son definidas como los puntos que tonalmente constituyen un límite y que son indicadas con un número "dependiendo del grado de transición que se percibe entre los bordes de cada par de palabras y entre la última palabra y el silencio final del enunciado" (Ortiz-Lira, 1999, p. 432). Asimismo, Cid y Ortiz-Lira (1998), al analizar la conducta prosódica de la función vocativo en el enunciado en posición inicial, central y final, buscan indagar respecto de "(a) la forma de la curva tonal del enunciado que contiene el vocativo, (b) la localización de los acentos tonales responsables de otorgar prominencia, (c) la distribución de la pausa y (d) la organización de los enunciados en grupos entonacionales" (Cid y Ortiz-Lira, 1998, p. 144). Este objetivo los lleva a darle prioridad a la presencia o ausencia de acentos tonales o sílabas prominentes como los indicadores de límites de separación, y a considerar la pausa solo como uno de los diversos criterios que permite demarcar grupos entonacionales y no como el único. Cid y Maluenda (2005), tras el objetivo de caracterizar el alargamiento segmental y sus distintas manifestaciones en el habla pública de Chile, propusieron una taxonomía que buscó dar cuenta del comportamiento prosódico de las pausas llenas y el valor expresivo, pragmático y discursivo en los que se expresa información no verbal al interior de los actos de habla. Sin embargo, los datos entregados se basan en una ejemplificación en la que buscan exponer el contorno entonacional de algunos enunciados de acuerdo con la función que el alargamiento cumple según el contexto, sin dar cuenta de tipos de pausa. En otro estudio, Cid y Céspedes (2008) tienen como objetivo realizar un análisis descriptivo del habla semiespontánea de 8 hombres y 8 mujeres provenientes de las localidades rulares de Culiprán y Popeta; en este trabajo, con el fin de diferenciar esta habla del habla estándar del país, proponen una caracterización de los aspectos fonotácticos (elisiones, asimilaciones, sinalefas, sinéresis, sustituciones) y discursivos (la presencia y recurrencia de pausas llenas o vacías, las partidas falsas, las repeticiones, el alargamiento segmental) más recurrentes de esta variedad dialectal. Sin embargo, su interés por esbozar una descripción desde una perspectiva fonofonológica da como resultado una enumeración de pocos ejemplos de rasgos fonoácticos desligados de su contexto de uso y producción. Asimismo, aunque propusieron inicialmente hablar acerca de las pausas como elemento discursivo relevante en el habla, no se menciona nada acerca de ellas en esta variedad dialectal, debido a que el análisis se centra en el alargamiento segmental vocálico y consonántico. Por último, Pereira (2007) intenta determinar el patrón prosódico subyacente de las oraciones ambiguas cuya cláusula de relativo corresponde al núcleo del complemento directo de la oración principal, es decir, con patrón de adjunción alta. Tras ese objetivo, la investigación se dividió en dos fases, la primera correspondió a la determinación del lugar de la pausa y del contorno melódico de 120 oraciones ambiguas ( 60 con patrón de adjunción alta y 60 con patrón 
de adjunción baja) emitidas por 10 estudiantes universitarios (4 hombres y 6 mujeres) nacidos en Concepción. La segunda fase consistió en un estudio perceptual para confirmar que el patrón prosódico de los enunciados emitidos generaba la interpretación esperada. Asimismo, se propuso una categorización de las pausas para verificar si la ubicación de la misma resultaba relevante en la producción de enunciados ambiguos. Finalmente, se llegó a la conclusión de que la pausa no constituye un elemento indispensable para la desambiguación de los enunciados. Sin embargo, el lugar y la duración de esta sí pueden determinar diferencias relevantes, porque los enunciados con adjunción baja requieren siempre de una pausa más larga que la usada en los enunciados con adjunción alta. En suma, la combinación de tonos y el cambio en la altura tonal en el contorno son los elementos que realmente permiten la desambiguación de enunciados con adjunción alta de la cláusula de relativo y no la presencia o ausencia de pausa.

En resumen, aunque existen descripciones parciales sobre ciertos fenómenos prosódicos en regiones específicas, tales como Santiago y Concepción, es evidente la ausencia de estudios interesados en proporcionar datos sobre las pausas en el español chileno, que enmarquen su objeto de estudio en un tipo específico de enunciado y en una situación comunicativa espontánea dada.

En este marco, el presente estudio se propone: a) dar cuenta de los mecanismos prosódicos con que los hablantes profesionales chilenos de dos ciudades extremas del país (Iquique y Punta Arenas) realizan pausas cuando producen oraciones subordinadas sustantivas objetivas en un discurso de orientación argumentativa en una situación comunicativa semiespontánea, b) determinar si se manifiestan diferencias en los procedimientos utilizados por estos hablantes cuando realizan pausas, considerando la variable de complejidad oracional, c) determinar si se manifiestan diferencias en los procedimientos utilizados por estos hablantes cuando producen pausas, considerando la variable género, y d) determinar si se manifiestan diferencias en los procedimientos utilizados por estos hablantes cuando realizan pausas, considerando la variable de procedencia geográfica.

\section{METODOLOGÍA}

\subsection{TIPO DE ESTUDIO}

Esta investigación constituye un estudio exploratorio y descriptivo en torno a las pausas silenciosas y a las pausas orales (alargamientos y vacilaciones) halladas al interior de 100 oraciones subordinadas sustantivas objetivas emitidas por hablantes provenientes de Iquique y de Punta Arenas. Asimismo, como estudio descriptivo, se detallan las características encontradas al interior del corpus y se proponen generalizaciones que pretenden dar cuenta de las pausas, relacionando estos resultados con la frecuencia de estas manifestaciones sonoras, el tipo de enunciado, el género de los informantes y su procedencia geográfica. 


\subsection{LOS INFORMANTES}

En la investigación, se consideraron 37 informantes, 19 hablantes de Iquique (10 mujeres, 9 hombres) y por 18 hablantes de Punta Arenas ( 9 mujeres, 9 hombres). Todos los sujetos observados eran personas con estudios profesionales completos.

\subsection{EL CORPUS}

El corpus de habla pertenece al proyecto Fondecyt $N^{o} 1120886$, y está constituido por un registro visual y auditivo de varias entrevistas y lecturas en voz alta perteneciente a una muestra representativa de hablantes profesionales chilenos. Es un registro oral socialmente aceptado en situaciones cotidianas de comunicación, caracterizado por una relativa espontaneidad, naturalidad y falta de planificación (Briz, 1996). Además, a causa de su naturaleza semiespontánea, permite caracterizar diversas realizaciones lingüísticas e indagar acerca de variabilidad dialectal y sociodialectal, considerando cuatro de los criterios de la propuesta del grupo Val.Es.Co (Hidalgo, 1997), para la caracterización del registro coloquial: "relación de igualdad, relación vivencial de proximidad, marco de interacción familiar y temática no especializada" (Hidalgo, 1997, p. 14), mecanismos con los que se busca garantizar mayor naturalidad y espontaneidad en el discurso. Como la población requerida consideraba personas con estudios superiores completos, la situación comunicativa de la entrevista planteó la interacción de un papel social (estrato sociocultural-profesión) y un papel funcional (relación simétrica) mediante un diálogo entre dos hablantes profesionales (nexo de igualdad). Asimismo, a pesar de no poder garantizar el conocimiento mutuo de los interlocutores, se procuró que la relación vivencial de proximidad se diera mediante el vínculo de un tercero, para de esta forma neutralizar en parte un desconocimiento total entre interlocutores que pudiese afectar tajantemente la actuación lingüística de los hablantes. Por otra parte, tras el objetivo de asegurar un marco de interacción familiar, los entrevistadores buscaron que el entorno físico en donde se desarrollaran las conversaciones estimulara la tranquilidad y comodidad de los informantes, considerando que, cuanto más familiar les resultara el espacio, mayor era la probabilidad de que la conversación fuese más espontánea y natural. Por consiguiente, los entrevistadores se desplazaron a los sitios de trabajo de los informantes e, incluso, algunos de ellos visitaron sus hogares. En adhesión a esto, desde el inicio de la entrevista se intentó generar una atmósfera cordial que disminuyera la tensión y a la vez permitiera conocer los intereses del informante.

Respecto de la entrevista propiamente tal, se buscó una interlocución que se diera cara a cara, una conversación sin predeterminación de turnos de habla ni planificación previa, de modo que se asegurara la espontaneidad y el carácter inmediato del diálogo. Aunque se realizaron entrevistas estructuradas en cuatro etapas (familiarización, descripción, argumentación y narración), se intentó estimular una 
alternancia sucesiva de intervenciones con el objetivo de que el informante conversara con naturalidad y, de este modo, se pudieran evitar silencios prolongados, alargar la actuación lingüística del informante o introducir nuevos temas, por parte del entrevistador, cuando se percataba de que los otros se iban agotando (López, 1994). Para ello, cada entrevistador trató de suscitar un método de retroalimentación con el fin de estimular una mayor producción lingüística.

En la entrevista, diseñada en 4 etapas, se buscó que su temática estuviera al alcance de cualquier individuo, por lo que cada parte incentivó la producción de un tipo de discurso específico sin relacionarlo con una temática especializada. En la primera, se incentivó una atmósfera familiar que permitiera el inicio de un diálogo tranquilo y relajado. En la segunda etapa se planteó como objetivo estimular las tres operaciones básicas de la descripción: la identificación del objeto o tema a describir, la enumeración de las partes o aspectos del objeto y la caracterización de las partes como del objeto mismo (Álvarez, 1996). En la tercera, en la que se centra el interés en este estudio, se pedía la opinión del informante acerca de un tema controversial que admitía distintos puntos de vista, con el objetivo de incentivar una valoración, un enfoque particular o la fundamentación de un juicio en torno a alguno de los temas planteados en la entrevista, escogido aleatoriamente por el entrevistador: la inscripción automática y el voto voluntario, las movilizaciones estudiantiles y la donación de órganos. En la cuarta etapa se pretendió que cada informante construyera un relato de su diario vivir y, una vez que lo hiciera, se le solicitaba que mencionara alguna anécdota que le hubiera sucedido en su trabajo o en su vida personal en la que se hubiese sentido amenazado o en la que alguna de las personas implicadas en la historia hubiera estado en grave peligro.

En cuanto a las condiciones técnicas de la grabación, esta se realizó con una grabadora digital TASCAM modelo DR-40, y la filmación con una cámara de video Sony modelo HDR-PJ230.

\subsection{SELECCIÓN DE LAS MUESTRAS DE HABLA}

Una vez recogido el corpus se seleccionó la parte de la entrevista que mejor representara un estilo de habla espontáneo, y que contara con fragmentos de habla continuos y estructurados. De este modo, se extrajo el pasaje de la entrevista en la que el entrevistador motivó que cada informante manifestara una posición determinada frente al objeto y defendiera, con razonamientos lógicos o enunciados argumentativos, su postura en relación con el mismo. Esta parte, ubicada en el momento central de la conversación, se ajustó perfectamente a los intereses del estudio, porque, a pesar de presentar rasgos propios del discurso oral, permitió identificar tres tipos de estructuras mayormente constantes: oraciones coordinadas adversativas, oraciones subordinadas sustantivas objetivas y oraciones subordinadas adverbiales causales. Sin embargo, luego de un análisis preliminar, se observó que las estructuras que complementaban verbos que expresan pensamiento y juicio (admitir, aprobar, calcular, considerar, creer, concluir, pensar, suponer, entender, criticar, etc.) fueron las más representativas de este 
tipo de discurso y permitían contar con un corpus de análisis suficiente para observar el comportamiento de los tipos de pausas en relación con las variables definidas.

De esta forma, el corpus de habla analizado en esta investigación quedó compuesto por 100 emisiones enunciadas por los 37 informantes. Con el propósito de favorecer el análisis de las pausas en oraciones de similar naturaleza gramatical, los enunciados se dividieron en dos grupos: el primero en el que se consideraron oraciones subordinadas sustantivas objetivas de un período subordinado, del tipo Creo / que mañana lloverá, y oraciones subordinadas sustantivas objetivas de dos períodos subordinados, en el que el segundo se relaciona con el primer período subordinado, del tipo Creo / que mañana lloverá / porque está nublado. En cada ciudad, se analizaron 36 oraciones subordinadas sustantivas objetivas de un período (18 en los hombres y 18 en las mujeres) y 14 oraciones subordinadas sustantivas objetivas de dos períodos ( 7 en los hombres y 7 en las mujeres).

\subsection{PROCEDIMIENTO DE ANÁLISIS}

Una vez leídas las transliteraciones de las entrevistas de las dos ciudades consideradas en este estudio, se procedió a la audición de cada registro en dos fases. En la primera fase se escuchó el fragmento de la entrevista que correspondía a la argumentación con el objetivo de conseguir familiarizarse con las características generales de la muestra oral, tanto a nivel prosódico como discursivo. En la segunda fase se seleccionó cada fragmento, prestando principal atención a todas las oraciones subordinadas sustantivas objetivas que se manifestaron en el pasaje argumentativo, las que fueron extraídas y transliteradas en su totalidad. Sin embargo, la realidad oral o discursiva, caracterizada por diversos elementos fonológicos como interrupciones, suspensiones, segmentación oracional, causal, etcétera, obligó a determinar criterios específicos que permitieran homologar la muestra de habla. Así, hubo que descartar las oraciones demasiado extensas, que mostraban una gran cantidad de frases $u$ oraciones intercaladas. Luego, las emisiones seleccionadas se dividieron en los dos grupos anteriormente mencionados. Una vez seleccionados los enunciados de cada ciudad considerada, se procedió a la audición detallada de cada fragmento extraído, marcando las pausas silentes y las pausas llenas presentes en cada enunciado. En relación con estas últimas, se consideraron en este tipo los alargamientos vocálicos y consonánticos, y las vacilaciones, expresadas mediante la repetición de una palabra o sílaba, el cambio de una palabra, etc. Finalmente se construyó una tabla que permitiese comparar los tipos de pausas considerando las variables enunciadas anteriormente. Los Anexos 1, 2, 3 y 4 muestran todas las oraciones analizadas, con las marcas definidas para diferenciar las pausas silentes, los alargamientos y las vacilaciones, considerando los dos tipos de oraciones, así como el género y la procedencia geográfica de los hablantes. 


\section{RESULTADOS}

\subsection{TIPOS DE PAUSA (CORPUS COMPLETO)}

Como es posible apreciar en el gráfico de la Figura 1, las pausas vacías o silencios son los que más se manifestaron en los enunciados analizados, alcanzando $49 \%$ del total de los tipos de pausas (81 casos), porcentaje que prácticamente dobla a los alargamientos y vacilaciones, los que alcanzan $23 \%$ (39 casos) y $28 \%$ (46 casos), respectivamente. De este modo, las pausas vacías o silencios constituyen el procedimiento más utilizado por los hablantes observados en este estudio.

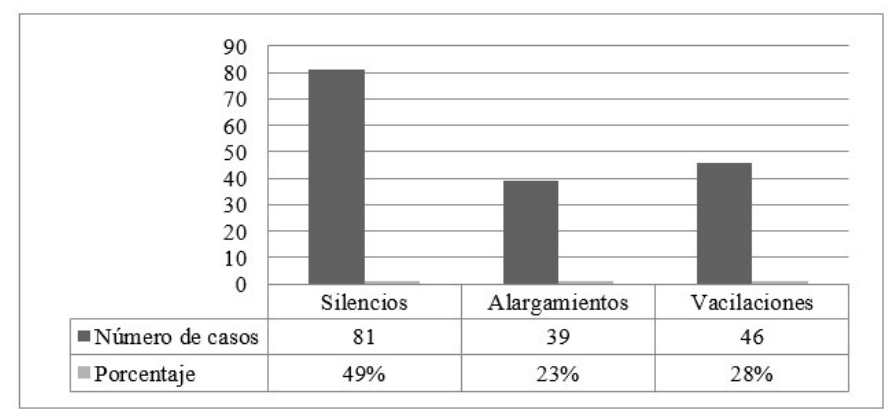

Figura 1. Tipos de pausas, el número de casos hallados tanto en la ciudad de Iquique como en la ciudad de Punta Arenas, en los 100 enunciados analizados.

\subsection{TIPOS DE PAUSAS EN ESTRUCTURAS DE UN PERÍODO $V S$. DOS PERÍODOS}

La Tabla 1 y los gráficos de las Figuras 2 y 3 muestran que, en los enunciados de un período, el grupo completo de informantes realizó 93 pausas, en tanto que en los enunciados de dos períodos los mismos informantes produjeron 73 pausas. Esta diferencia de apenas 20 pausas es muy relevante, sobre todo si se tiene en cuenta que los enunciados de un período son 72 ( 18 por cada uno de los 4 grupos considerados) y 28 los de dos períodos (7 por cada uno de los 4 grupos de la muestra total). De este modo, si se hubiese contado con más enunciados de dos períodos, sin duda, la cantidad de pausas de los tres tipos definidos habría aumentado de manera notable $\mathrm{y}$, muy probablemente, si se sigue la misma tendencia observada, las pausas en estructuras oracionales de dos períodos se habrían duplicado. Téngase en cuenta respecto de lo anterior que si se hubiese contado con 18 oraciones de dos períodos en cada uno de los cuatro grupos considerados, entonces el promedio de 2,6 pausas por enunciado, extraído de las 28 oraciones analizadas, alcanzaría 187 pausas $(2,6 \times 72=187)$. Ello, además, resulta predecible, porque las estructuras sintácticas más complejas deberían generar un mayor número de pausas, independientemente del tipo que se produzcan.

Por otra parte, en las oraciones de un período, los tipos de pausas se distribuyen más o menos regularmente: el mayor porcentaje lo obtienen los silencios con $39 \%$ de 
las pausas analizadas (36 casos), le siguen las vacilaciones con 34\% (32 casos) y los alargamientos con $27 \%$ ( 25 casos). En las oraciones de dos períodos, en cambio, los silencios alcanzan $62 \%$ de las pausas observadas (45 casos), en tanto que las vacilaciones y los alargamientos coinciden en 19\% (14 casos para cada uno de los tipos de pausas). Lo anterior quiere decir que los hablantes frente a estructuras sintácticas más complejas tienden a producir más pausas silenciosas que a alargar la secuencia o a vacilar mientras hablan.

Tabla 1. Un período $v s$. dos períodos

\begin{tabular}{|l|c|c|c|c|c|}
\hline Tipo de pausa & \multicolumn{2}{|c|}{$\begin{array}{c}\text { Un período } \\
(72 \text { oraciones })\end{array}$} & \multicolumn{2}{c|}{$\begin{array}{c}\text { Dos períodos } \\
(28 \text { oraciones })\end{array}$} & Total \\
\hline Silencios & 36 & $39 \%$ & 45 & $62 \%$ & 81 \\
\hline Alargamientos & 25 & $27 \%$ & 14 & $19 \%$ & 39 \\
\hline Vacilaciones & 32 & $34 \%$ & 14 & $19 \%$ & 46 \\
\hline \multicolumn{1}{|c|}{93} & \multicolumn{2}{|c|}{73} & 166 \\
\hline
\end{tabular}

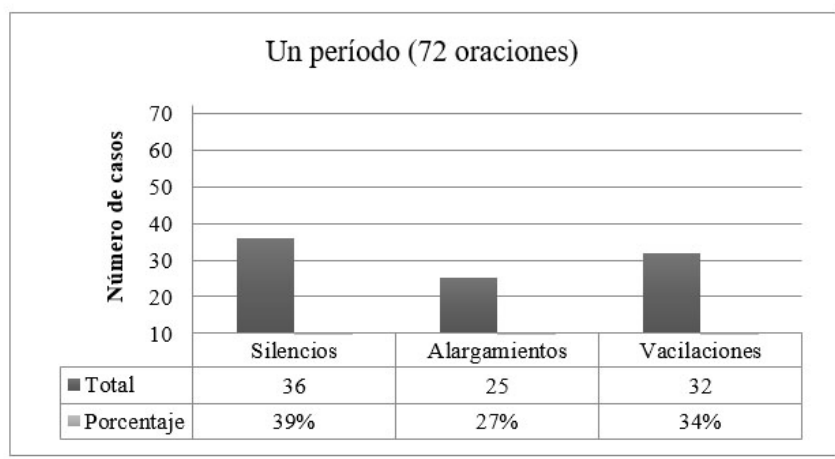

Figura 2. Un período vs. dos períodos

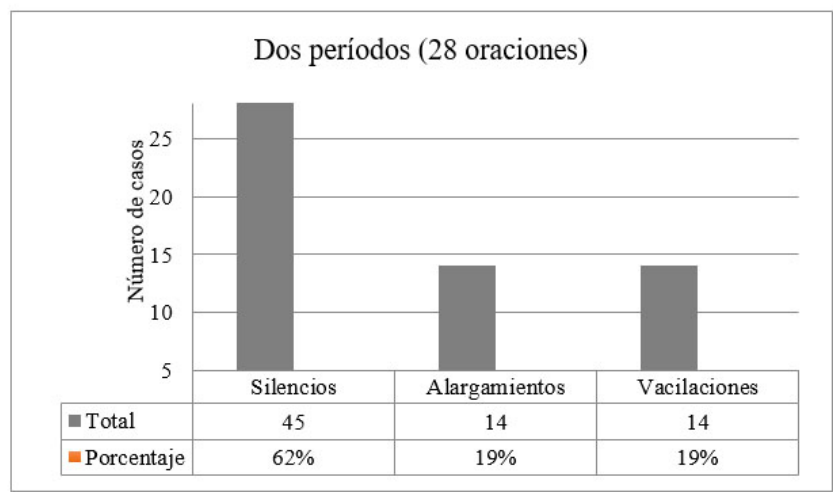

Figura 3. Un período $v s$. dos períodos 
Al explorar la asociación entre la variable independiente (tipo de pausa) y la variable dependiente (complejidad oracional) mediante la realización de una prueba Chi-cuadrado, se determinó la existencia de una asociación estadísticamente significativa entre ambas variables en cuanto a la frecuencia de aparición de los tipos de pausas al interior de los enunciados bajo estudio $(\chi 2=8,865, \mathrm{df}=2, \mathrm{p}$-value $=0,01188$; $\mathrm{V}$ de Cramer $=0,231$ ) con V de Cramer que indica un efecto moderado (Kotrlik, Williams \& Jabor, 2011).

\subsection{TIPOS DE PAUSAS EN RELACIÓN CON LA VARIABLE GÉNERO}

La Tabla 1 y los gráficos de las Figuras 2 y 3 muestran, con total claridad, que en los tipos de pausas considerados en este trabajo los hombres tienden a realizar un mayor número de pausas. En efecto, en relación con los silencios, los hombres produjeron 41 casos (51\%) en tanto que las mujeres presentaron 40 casos (49\%). En cuanto a los alargamientos, esta diferencia aumentó, pues los hombres produjeron 25 casos (64\%), en tanto que las mujeres presentaron 14 casos $(36 \%)$. Por último, en relación con las vacilaciones, nuevamente los hombres produjeron más casos de este tipo de pausas con 30 alargamientos $(65 \%)$, en contraste con las mujeres que presentaron 16 casos $(35 \%)$. Por lo mismo, los resultados generales, según la variable género, demuestran que los hombres produjeron 96 casos (58\%) y las mujeres 70 casos (42\%).

Tabla 2. Comparación de los tipos de pausas en mujeres y hombres

\begin{tabular}{|l|c|c|c|c|}
\hline \multicolumn{1}{|c|}{ Tipo de pausa } & \multicolumn{2}{|c|}{ Mujeres } & \multicolumn{2}{c|}{ Hombres } \\
\hline Silencios & 40 & $49 \%$ & 41 & $51 \%$ \\
\hline Alargamientos & 14 & $36 \%$ & 25 & $64 \%$ \\
\hline Vacilaciones & 16 & $35 \%$ & 30 & $65 \%$ \\
\hline \multicolumn{1}{|c|}{ Total } & 70 & $42 \%$ & 96 & $58 \%$ \\
\hline
\end{tabular}

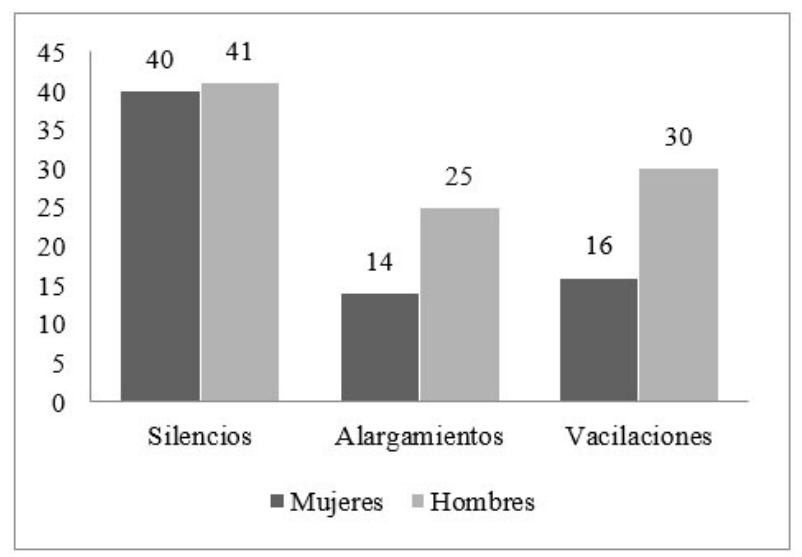

Figura 4. Comparación de los tipos de pausas en mujeres y hombres 
No obstante, la realización de una prueba de Chi-cuadrado en la que la variable dependiente es el fenómeno analizado y la dependiente, el género de los informantes demostró que no hay una asociación estadísticamente significativa con relación a la frecuencia de aparición de los tres tipos de pausa al interior de los enunciados $(\chi 2=3,387$, $\mathrm{df}=2, \mathrm{p}$-value $=0.1839 ; \mathrm{V}$ de Cramer $=0,143)($ Kotrlik, Williams \& Jabor, 2011).

\subsection{TIPOS DE PAUSAS EN RELACIÓN CON LA VARIABLE GÉNERO EN ORACIONES DE UN PERÍODO}

Se consideró pertinente observar si los hallazgos encontrados en relación con las pausas y el efecto de la variable género se mantenían en oraciones de uno o dos períodos.

Así, la Tabla 3 y el gráfico de la Figura 5 muestran, de manera contundente, que los tipos de pausas observados en este estudio mantenían la tendencia determinada en los resultados generales en los tres tipos de pausas observados. En oraciones de un período, respecto de los silencios, los hombres produjeron 21 casos (58\%) y las mujeres presentaron 15 casos (42\%); en relación con los alargamientos, los hombres produjeron 17 casos $(68 \%)$, en tanto que las mujeres presentaron 8 casos (32\%); por último, en cuanto a las vacilaciones, los hombres produjeron 20 casos (38\%), en tanto que las mujeres presentaron 12 casos (62\%). Si se observan, en consecuencia, el total de resultados en oraciones de un período, se comprueba no solo que la tendencia de los resultados generales se mantuvo, sino que también se puede apreciar que las diferencias aumentan, pues los hombres produjeron 58 pausas (62\%), cuatro puntos porcentuales más, en oposición a las mujeres que presentaron 35 pausas $(38 \%)$, cuatro puntos porcentuales menos.

Tabla 3. Comparación de mujeres y hombres en oraciones de un período

\begin{tabular}{|l|c|c|c|c|}
\hline \multicolumn{5}{|c|}{ Oraciones de un período } \\
\hline Tipo de pausa & \multicolumn{2}{|c|}{ Mujeres } & \multicolumn{2}{c|}{ Hombres } \\
\hline Silencios & 15 & $42 \%$ & 21 & $58 \%$ \\
\hline Alargamientos & 8 & $32 \%$ & 17 & $68 \%$ \\
\hline Vacilaciones & 12 & $38 \%$ & 20 & $62 \%$ \\
\hline Total & 35 & $38 \%$ & 58 & $62 \%$ \\
\hline
\end{tabular}

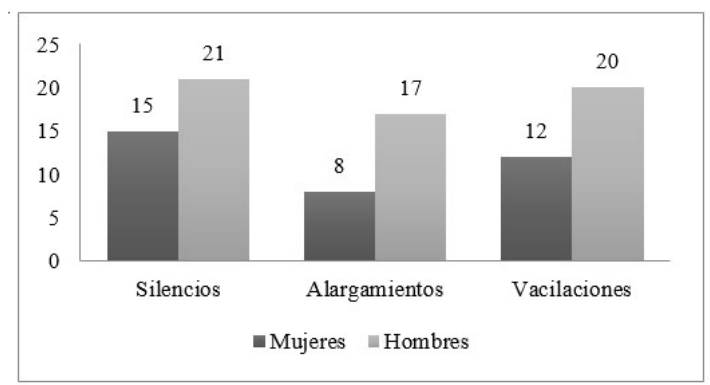

Figura 5. Comparación de mujeres y hombres en oraciones de un período 
Al igual que en los anteriores análisis estadísticos, el valor de significación estadística con relación a la frecuencia de aparición de los tres tipos de pausa al interior de los enunciados de un período emitidos tanto por mujeres como por hombres indicó que globalmente no hay diferencias significativas $\left(\chi^{2}=0,5878, \mathrm{df}=2\right.$, $\mathrm{p}$-value $=$ 0,7454; V de Cramer = 0,079) (Kotrlik, Williams \& Jabor, 2011)

\subsection{TIPOS DE PAUSAS EN RELACIÓN CON LA VARIABLE GÉNERO EN ORACIONES DE DOS PERÍODOS}

En cuanto a las oraciones de dos períodos y la relación con la variable género, la Tabla 4 y el gráfico de la Figura 6 muestran, en general, las mismas tendencias observadas en las oraciones de un período, aunque con una leve variación. Efectivamente, en los silencios, son las mujeres las que produjeron más casos, con un total de 25 pausas de este tipo (56\%), en tanto los hombres presentaron 20 pausas silenciosas (44\%). Con todo, la tendencia se recupera con los alargamientos, pues los hombres realizaron 8 casos $(57 \%)$, mientras que las mujeres presentaron 6 casos (43\%), así como también con las vacilaciones, tipos de pausas que muestran diferencias más marcadas, con 10 pausas de este tipo en los hombres (71\%), en oposición a las mujeres que produjeron apenas 4 vacilaciones $(29 \%)$. Los resultados totales de las oraciones de dos períodos muestran una menor diferencia entre hombres y mujeres, con 38 pausas producidas por los primeros (52\%), en contraste con las 35 pausas generadas por las segundas (48\%). En todo caso, a causa de que hubo un tipo de pausa que invirtió la tendencia bastante regular observada anteriormente, no es extraño que, en las pausas de dos períodos, se haya manifestado un acercamiento en la producción de pausas entre hombres y mujeres.

Tabla 4. Comparación de mujeres y hombres en oraciones de dos períodos

\begin{tabular}{|l|c|c|c|c|}
\hline \multicolumn{4}{|c|}{ Oraciones de dos períodos } \\
\hline Tipo de pausa & \multicolumn{2}{|c|}{ Mujeres } & \multicolumn{2}{c|}{ Hombres } \\
\hline Silencios & 25 & $56 \%$ & 20 & $44 \%$ \\
\hline Alargamientos & 6 & $43 \%$ & 8 & $57 \%$ \\
\hline Vacilaciones & 4 & $29 \%$ & 10 & $71 \%$ \\
\hline Total & 35 & $48 \%$ & 38 & $52 \%$ \\
\hline
\end{tabular}

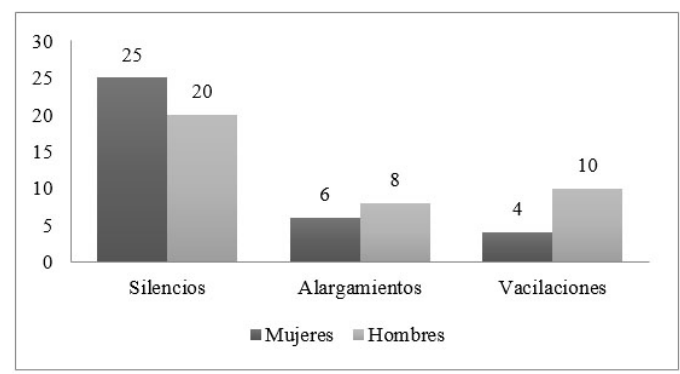

Figura 6. Comparación de mujeres y hombres en oraciones de dos períodos 
Con la finalidad de evaluar la asociación con significatividad estadística entre las variables anteriormente mencionadas, se ejecutó el mismo análisis anterior mediante la aplicación de la prueba de Chi-cuadrado. Los resultados indicaron que otra vez no hay diferencias significativas entre ambas variables en cuanto a la frecuencia de aparición de los tipos de pausas al interior de los enunciados de dos períodos emitidos por informantes de ambos géneros $(\chi 2=3,295, \mathrm{df}=2$, $\mathrm{p}$-value $=0,1925 ; \mathrm{V}$ de Cramer $=$ 0,212) (Kotrlik, Williams \& Jabor, 2011).

\subsection{TIPOS DE PAUSAS EN RELACIÓN CON LA VARIABLE DE PROCEDENCIA GEOGRÁFICA}

Tras el objetivo de determinar si la incidencia de la procedencia geográfica del hablante en la configuración prosódica de una de las estructuras más frecuentemente empleada en el discurso con intención argumentativa, se compararon de manera directa ambos grupos. La Tabla 5 y el gráfico de la Figura 7 muestran, de modo claro y contundente, que en los tres tipos de pausas consideradas en este trabajo los hablantes de Iquique tienden a realizar un mayor número de hesitaciones. En efecto, en relación con los silencios, los hablantes iquiqueños produjeron 47 casos (58\%), en tanto que los informantes puntarenenses presentaron 34 casos (42\%). En cuanto a los alargamientos, esta diferencia disminuyó levemente, pues los hablantes de Iquique produjeron 22 casos (56\%), en tanto que los informantes de Punta Arenas presentaron 17 casos (44\%). Por último, en relación con las vacilaciones, otra vez, los hablantes iquiqueños produjeron más casos de este tipo de pausas con 29 alargamientos (63\%), en contraste con los informantes puntarenenses que presentaron 17 casos (37\%). De acuerdo con lo anterior, los resultados que consideran la totalidad de pausas, según la variable de procedencia geográfica, indican que los iquiqueños produjeron 98 hesitaciones (59\%), mientras que los puntarenenses presentaron 68 hesitaciones (41\%), valores que confirman la tendencia observada en cada uno de los tipos de pausas analizadas.

Tabla 5. Comparación entre zonas geográficas (Iquique y Punta Arenas)

\begin{tabular}{|l|c|c|c|c|}
\hline Tipo de pausa & \multicolumn{2}{|c|}{ Iquique } & \multicolumn{2}{c|}{ Punta Arenas } \\
\hline Silencios & 47 & $58 \%$ & 34 & $42 \%$ \\
\hline Alargamientos & 22 & $56 \%$ & 17 & $44 \%$ \\
\hline Vacilaciones & 29 & $63 \%$ & 17 & $37 \%$ \\
\hline Total & 98 & $59 \%$ & 68 & $41 \%$ \\
\hline
\end{tabular}




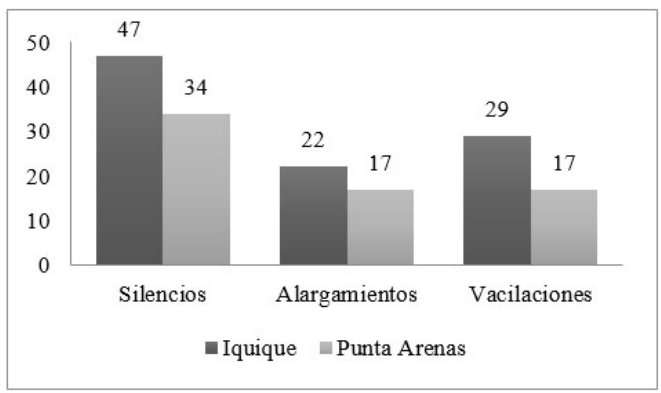

Figura 7. Comparación entre zonas geográficas (Iquique y Punta Arenas)

Al explorar la asociación entre la variable independiente (tipo de pausa) y la variable dependiente (procedencia geográfica) mediante la ejecución de una prueba Chi-cuadrado, nuevamente indicó que de manera global no hay diferencias significativas $(\chi 2=0,4509, \mathrm{df}=2, \mathrm{p}$-value $=0,7981$; V de Cramer $=0,052)($ Kotrlik, Williams \& Jabor, 2011).

\subsection{TIPOS DE PAUSAS EN RELACIÓN CON LA VARIABLE DE PROCEDENCIA GEOGRÁFICA EN ORACIONES DE UN PERÍODO}

En este caso se consideró también pertinente observar si los hallazgos encontrados en relación con las pausas y el efecto de la variable de procedencia geográfica se mantenían en oraciones de uno o dos períodos.

En oraciones de un período, la Tabla 6 y el gráfico de la Figura 8 muestran, nuevamente de manera clara y contundente, que los hablantes de Iquique produjeron más pausas en los tres tipos observados que los hablantes de Punta Arenas. Respecto de los silencios, los informantes iquiqueños presentaron 22 casos (61\%) mientras que los hablantes de Punta Arenas produjeron 14 casos (39\%). En cuanto a los alargamientos, la diferencia disminuyó levemente, pues los informantes iquiqueños presentaron 14 casos $(56 \%)$, en tanto que los hablantes puntarenenses produjeron 11 casos $(44 \%)$. Finalmente, en relación con las vacilaciones, la diferencia otra vez aumentó, ya que los informantes de Iquique presentaron 19 casos (59\%), en tanto que los hablantes de Punta Arenas produjeron 13 casos (41\%). Por ello, al observar el total de resultados considerando la variable de procedencia geográfica en oraciones de un período, se corrobora la tendencia general indicada en el apartado anterior, esto es, los iquiqueños presentan más pausas con 55 hesitaciones (59\%), mientras que los puntarenenses produjeron solamente 38 pausas (41\%). 
Tabla 6. Comparación entre zonas geográficas (Iquique y Punta Arenas)

\begin{tabular}{|l|c|c|c|c|}
\hline \multicolumn{4}{|c|}{ Oraciones de un período } \\
\hline Tipo de pausa & \multicolumn{2}{|c|}{ Iquique } & \multicolumn{2}{c|}{ Punta Arenas } \\
\hline Silencios & 22 & $61 \%$ & 14 & $39 \%$ \\
\hline Alargamientos & 14 & $56 \%$ & 11 & $44 \%$ \\
\hline Vacilaciones & 19 & $59 \%$ & 13 & $41 \%$ \\
\hline \multicolumn{1}{|c|}{ Total } & 55 & $59 \%$ & 38 & $41 \%$ \\
\hline
\end{tabular}

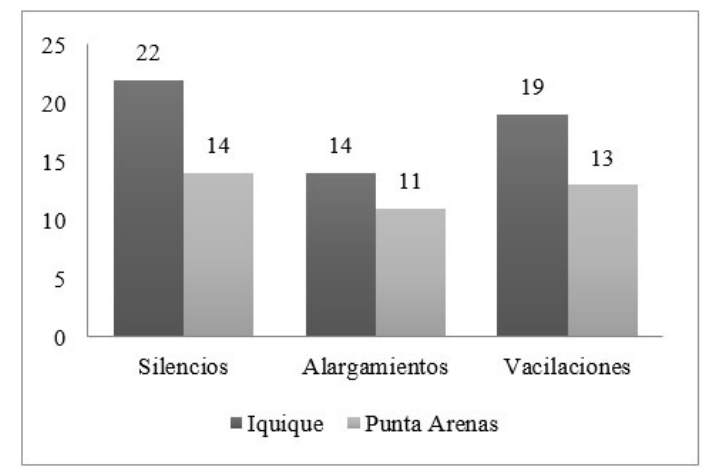

Figura 8. Comparación entre zonas geográficas (Iquique y Punta Arenas)

No obstante, el valor de significación de una prueba de Chi-cuadrado, en la que la variable independiente (tipo de pausa) y la variable dependiente (procedencia geográfica) en oraciones de un período, indicó otra vez la inexistencia de diferencias significativas $\left(\chi^{2}=0,16062, \mathrm{df}=2, \mathrm{p}\right.$-value $=0,9228 ; \mathrm{V}$ de Cramer $\left.=0,042\right)($ Kotrlik, Williams \& Jabor, 2011).

\subsection{TIPOS DE PAUSAS EN RELACIÓN CON LA VARIABLE DE PROCEDENCIA GEOGRÁFICA EN ORACIONES DE DOS PERÍODOS}

Respecto de las oraciones de dos períodos y la relación con la variable de procedencia geográfica de los hablantes, los resultados de la Tabla 7 y el gráfico de la Figura 9 vuelven a corroborar lo que se ha venido determinando anteriormente, esto es, los hablantes de Iquique presentaron más pausas que los hablantes de Punta Arenas, sin importar el tipo de pausas producidas. Efectivamente, en los silencios, los hablantes iquiqueños presentaron 25 casos (56\%), en tanto que los informantes puntarenenses produjeron 20 casos (44\%). En los alargamientos se observan porcentajes muy similares a los establecidos con los silencios, esto es, los hablantes de Iquique presentaron 8 casos (57\%) y los informantes de Punta Arenas 6 casos (43\%). La tendencia observada, que ya constituye un fenómeno sistemático, se verifica de manera más categórica con las vacilaciones, ya que los hablantes iquiqueños presentaron 10 casos (71\%), en tanto que los informantes puntarenenses produjeron solo 4 casos $(29 \%)$. De este modo, el total de 
pausas analizadas no hace más que confirmar la tendencia que se ha venido apreciando, vale decir, los hablantes de Iquique presentaron 43 pausas (59\%), mientras que los hablantes de Punta Arenas produjeron 30 pausas (41\%).

Tabla 7. Comparación entre zonas geográficas (Iquique y Punta Arenas)

\begin{tabular}{|l|c|c|c|c|}
\hline \multicolumn{4}{|c|}{ Oraciones de dos períodos } \\
\hline Tipo de pausa & \multicolumn{2}{|c|}{ Iquique } & \multicolumn{2}{c|}{ Punta Arenas } \\
\hline Silencios & 25 & $56 \%$ & 20 & $44 \%$ \\
\hline Alargamientos & 8 & $57 \%$ & 6 & $43 \%$ \\
\hline Vacilaciones & 10 & $71 \%$ & 4 & $29 \%$ \\
\hline Total & 43 & $59 \%$ & 30 & $41 \%$ \\
\hline
\end{tabular}

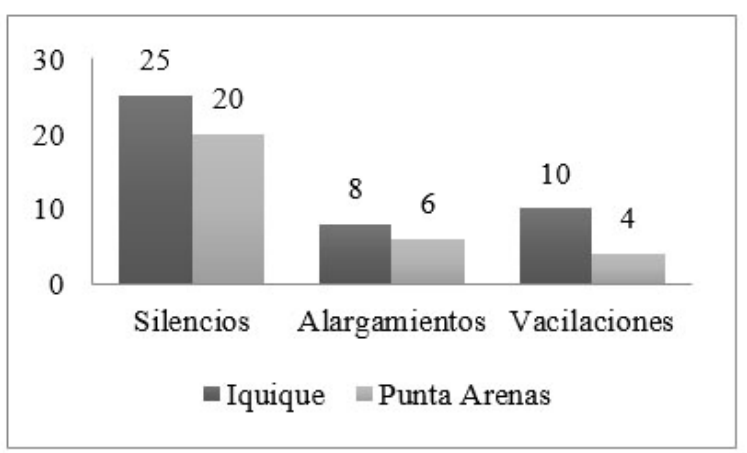

Figura 9. Comparación entre zonas geográficas (Iquique y Punta Arenas)

Siguiendo el mismo procedimiento anteriormente efectuado, se ejecutó la última prueba de Chi-cuadrado de los tipos de pausas en relación con la variable de procedencia geográfica en oraciones de dos períodos. Los resultados demostraron nuevamente que no hay una asociación estadísticamente significativa con relación a la frecuencia de aparición de los tres tipos de pausa al interior de las zonas geográficas en oraciones de dos períodos $(\chi 2=1,1336, \mathrm{df}=2, \mathrm{p}$-value $=0,5673 ; \mathrm{V}$ de Cramer $=0,125)$ (Kotrlik, Williams \& Jabor, 2011).

\section{CONCLUSIONES}

Si se consideran los objetivos de este trabajo, se puede afirmar que los cuatro propósitos declarados fueron cumplidos a cabalidad, pues se logró describir el comportamiento prosódico de tres tipos de pausas en el habla semiespontánea gracias a un riguroso diseño metodológico que permitió fusionar dos planos. Por un lado, equilibró un número similar de informantes tanto en Iquique como en Punta Arenas, y, por otro, posibilitó la observación de la oración subordinada sustantiva objetiva como la estructura sintáctica recurrente en un tipo discursivo que, si bien promueve la naturalidad de la expresión oral, demanda un compromiso cognitivo que requiere la 
ejecución de diversas estrategias mentales en las que el hablante diseña y planifica verbalmente su pensamiento, como lo es el discurso con intención argumentativa. Sin duda, las etapas consideradas en la investigación dan la necesaria confiabilidad a los resultados establecidos en la caracterización de las pausas en dos ciudades ubicadas geográficamente en zonas extremas del país.

Respecto de los resultados generales relacionados con los tipos de pausas, se constató la preferencia de los hablantes observados por el empleo de las pausas que les permitieran, durante breves lapsos de tiempo, detenerse en su discurso, con el objetivo de reestructurar las ideas sin perder su turno de habla, es decir, las pausas silenciosas. En contraste con estas, las pausas de carácter lexicalizado o semantizado, como los alargamientos y las vacilaciones, se presentaron en menor medida. Este hecho, por lo demás, se manifiesta en forma independiente al tipo de oración considerado, a la procedencia geográfica de los hablantes observados y al género de los informantes analizados.

En relación con las oraciones de un período subordinado comparadas con oraciones de dos períodos subordinados, se pudo apreciar que las primeras produjeron más pausas que las segundas. No obstante lo anterior, esta tendencia muy probablemente cambiaría si el número de oraciones de dos períodos subordinados hubiese sido el mismo que el de un período, es decir, 18 oraciones, y no 7 como finalmente ocurrió. En otras palabras, si el número de oraciones de dos períodos hubiera sido también 18, muy probablemente la cantidad de pausas superaría, por lejos, las cifras finalmente determinadas en este estudio.

En cuanto a los resultados de la variable género de los informantes, los resultados indicaron que los hombres producen más pausas que las mujeres, fenómeno que persistió, en general, en oraciones de uno y dos períodos. La excepción solo se observó respecto de las pausas silenciosas en oraciones de dos períodos, las que presentaron un número mayor de casos en las mujeres que en los hombres. De todas formas, los resultados indican que los hombres, cuando hablan, muestran tendencia a expresarse de manera menos fluida que las mujeres.

Finalmente, respecto de los resultados de las pausas asociados con la variable procedencia geográfica de los informantes observados, estos fueron más categóricos: sea en su totalidad o en oraciones de un período o de dos períodos, los hablantes de Iquique siempre produjeron más silencios, alargamientos y vacilaciones que los hablantes de Punta Arenas. Estos hechos indicarían, de manera sistemática, que los hablantes nortinos, cuando hablan, o se silencian o se entraban más que los hablantes del extremo sur.

Estos últimos datos sugieren, además, la posibilidad de seguir investigando qué sucede con las pausas en hablantes de otras ciudades del país, no solo porque se cuenta con un corpus de habla semiespontánea de otras zonas geográficas de Chile, el que reúne prácticamente las mismas características que las consideradas en Iquique y Punta Arenas, sino porque sería interesante observar qué ocurre con las pausas en ciudades de 
mayor densidad poblacional, como Concepción y Santiago. De hecho, se cuenta con datos preliminares que muestran que Concepción presenta más pausas que Iquique y Punta Arenas, y Santiago supera la cantidad de hesitasiones en relación con las tres ciudades anteriormente mencionadas.

\author{
Universidad de Los Andes* \\ Cra $1 N^{o} 18^{a}$-12. Código postal 111711, Bogotá (Colombia) \\ smhernandezr@gmail.com \\ Universidad de Concepción** \\ Departamento de Español \\ Casilla 160-C, Correo 3, Concepción (Chile) \\ jstot@udec.cl
}

\title{
OBRAS CITADAS
}

Álvarez, Gerardo (1996). Textos y discursos: introducción a la lingüística del texto. Concepción: Universidad de Concepción.

Barkat, Melissa; Gayraud, Frédérique (2009). "Pauses et hésitations dans le discours de patients Alzheimer et chez la personne a $\square$ gée saine", 3 Journées de Phonétique Clinique, Aix-en-Provence, 4-5 diciembre.

Blondet, María (2006). Variaciones de la velocidad de habla en español: patrones fonéticos y estrategias fonológicas. Un estudio desde la producción. Tesis doctoral. Mérida: Universidad de Los Andes.

Briz, Antonio (2001). El español coloquial en la conversación. Esbozo de pragmagramática. Barcelona: Ariel Lingüística.

Cicres, Jordi (2007). "Análisis discriminante de un conjunto de parámetros fonéticoacústicos de las pausas llenas para identificar hablantes", Síntesis Tecnológica $3(2), 87-98$.

Cruttenden, Alan (1990). Entonación: teoría general y aplicación al inglés. Barcelona: Teide.

Cid, Miriam; Céspedes, Macarena (2008) "Rasgos de simplificación en el habla rural de dos localidades de Chile: descripción fonotáctica y discursiva". Literatura y Lingüística 19, 197-210.

Cid, Miriam; Maluenda, Lorena (2005). "El alargamiento segmental en el habla pública de Chile: comportamiento prosódico-discursivo", Onomázein 11/1, 43-55.

Cid, Miriam; Ortiz-Lira, Héctor (1998). "La conducta prosódica del vocativo en el español culto de Santiago de Chile", Onomázein 3,143- 162.

Crystal, David (1971). "Relative and absolute in intonation analysis", Journal of the International Phonetic Association 1, 17-28.

Gussenhoven, Carlos (2002). "Phonology of Intonation", GLOT International vol. 6, 271-284. 
Hidalgo, Antonio (1997). Entonación coloquial. Función Demarcativa y Unidades de Habla. Valencia: ANEJO XXI de Cuadernos de Filología.

Kotrlik, Joe; Williams, Heather; Jabor, Khata (2011). "Reporting and Interpreting Effect Size in Quantitative Agricultural Education Research", Journal of Agricultural Education, número 52(1), 132-142.

López, Humberto (1994). Métodos de investigación lingüística. Salamanca: Ediciones del Colegio de España.

Machuca, María; Llisterri, Joaquín; Ríos, Antonio (2015). "Las pausas sonoras y los alargamientos en español: un estudio preliminar", Normas 5, 81-96.

Ortiz-Lira, Héctor (1999). "La aplicación de ToBI a un corpus del español de Chile". Onomazein 4, 429-442.

Pereira, Yasna (2007). "Determinación del patrón prosódico de las oraciones ambiguas con adjunción alta de la cláusula de relativo", Onomázein 16,139-158.

Tapia-Ladino, Mónica; Valdivieso, Humberto (2000). "Consideraciones para analizar los aspectos temporales de la producción del habla", Onomázein 5, 119-126.

Valdivieso, Humberto; Tapia-Ladino, Mónica; Quiroga, Pilar (2003). "Producción de unidades lingüísticas y no lingüísticas en el habla de pacientes con enfermedad de Alzheimer", Anales de psiquiatría, Vol. 19, número 8, 323-328.

\section{ANEXO 1}

Zona geográfica de Iquique

Enunciados de un período emitidos por mujeres iquiqueñas

1. Opino que es bueno.

2. Creo que ha sido súper poco (...) proba.

3. Siento en mis sensaciones que la gente se olvidó de eso.

4. Puede que no vayan a votar.

5. Creen que eso es (sss) atender la cultura de la gente.

6. Todo el mundo sabe que Soria va a salir.

7. Ellos creen que todavía se le puede seguir engañando a la gente.

8. Yo creo que (eee) (...) ahí está también (el desconten) el descontento de la gente.

9. No creo (que) que exista un gran cambio.

10. Pienso que también se ha ganado a su gente.

11. Veo que ahí es como muy similar.

12. Yo creo (...) que (eee) va a estar un poco peleada.

13. Pienso que facilita bastante (eee) el proceso.

14. Siento (que) que vamos a tener un dejavú.

15. No veo que haya interés (sss) real (...) en participar.

16. Creo que es el sistema correcto.

17. Encuentro que es un deber cívico ir a votar.

18. Yo creo que muchos jóvenes se van a restar. 
Enunciados de dos períodos emitidos por mujeres iquiqueñas.

1. Sé (...)que hay mucha gente(...) que votaría por ella y mucha gente que votaría por Soria.

2. (Creo) creo que es el sistema correcto(...) pero dudo profundamente del efecto(ooo) generado.

3. Creo que habrá alguien por ahí (iii) pero (no) no me queda tan claro.

4. Pienso que (...) cada uno tiene que (...) decidir si realmente (se) se siente identificado con alguien y emitir un voto.

5. A mí me parece positivo (...) primero que la inscripción sea automática y el voto $(. .$.$) voluntario.$

6. Creo que es necesario (...) pero (...) me falta fe.

7. A mí me interesa que Iquique (...) crezca (aaa) surja (...) sea una ciudad (aaa) (...) linda y segura.

Pausas vacías (silencios)

Pausas llenas (alargamientos)

Pausas llenas (vacilaciones)

Total

1 período
4
5
3
12

2 períodos

12

4

3

19

\section{ANEXO 2}

Zona geográfica de Iquique

Enunciados de un período emitidos por hombres iquiqueños

1. Yo creo que a la derecha (...) le conviene (...) generar un escenario político nuevo.

2. Pienso que la derecha (...) a lo mejor con eso quiere aumentar un poco (...) las posibilidades (de) de adhesión.

3. Me parece que ahí sí entra un poco en conflicto el (lll) (...) tema.

4. Recuerda que en ese tiempo (estaba) (eh) Pinochet todavía estaba vivo.

5. Fíjate que en este gobierno (...) todavía no se construye nada.

6. Me parece (que) que las alternativas son locales.

7. No creo que eso se traduzca (eh) (...) (en) en resultados $(d e)$ (...) de la inscripción automática.

8. Yo creo que es (...) está en (...) la pelea está (entre) (...) entre los dos de nuevo.

9. Fíjate que ninguno de los o por lo menos ninguno de los dos tiene la intención de (eee) (...) de ir a votar.

10. Parece que la juventud está un poco reacia (aaa) a participar (de esta) (aaa) de esta actividad.

11. Yo pienso que en otros países la situación es diferente.

12. La historia (...) diría (aaa) que va a salir (eh) (...) Jorge Soria.

13. Creo que va a aumentar la cantidad (de) (eee) de votantes. 
14. Yo creo que en el fondo la gente en la medida que sea (aaa) importante la elección va a participar.

15. Yo creo que se va a mantener un universo (...) (re) relativamente (...) parecido a las elecciones pasadas.

16. ( $\left.\mathrm{Si}^{\prime}\right)$ Siento yo digamos que son (eh) candidatos (oos) muy populistas.

17. Yo creo que (el) la juventud poco se va a inscribir.

18. Yo creo que de una u otra manera (...) eso generó cierta empatía en las (aas) (...) (en) en las mujeres sobre todo.

Enunciados de dos períodos emitidos por hombres iquiqueños

1. Yo espero que participen (...) pero (ooo) (no) no estoy muy seguro que así sea.

2. Siento digamos que (eh) la alcaldía (...) (eh) prácticamente es manejada (aaa) como una dictadura por él (...) porque tiene todo el poder.

3. Nosotros pensamos que iba a salir pero (ooo) (...) salió la Mirta.

4. No creo que $(l a)$ la inscripción automática (...) (eh) (...) vaya a tener un impacto en que (...) aumenten los votos $(d e)(. .$.$) de estas elecciones.$

5. Yo creo que eso generó (...) empatía en las mujeres (...) porque la mayoría de ellas viven así.

6. Yo creo que (...) es probable que tenga algún impacto en el número de votantes $(. .$.$) pero es marginal.$

7. Me parece que ahí sí entra en conflicto (ooo) (el) el tema porque (...) porque hoy día hay muchos chilenos en esa situación.

$$
1 \text { período } 2 \text { períodos }
$$

18

9

16

43
13

4

7

\section{ANEXO 3}

Zona geográfica de Punta Arenas

Enunciados de un período emitidos por mujeres puntarenences

1. Yo creo que no todavía.

2. Yo creo que eso (ooo) fue chocante para mí.

3. Hay que ver en el camino.

4. Tiene que tenerla en (een) (...) (eh) cómo se dice seguimiento.

5. Generalmente se dice que Magallanes es (una) (...) una región de (...) izquierda.

6. Entienda que (eee) (...) nosotros necesitamos (buenos) buenas autoridades.

7. Yo creo que hasta como marzo del próximo año.

8. Necesito(...) que me represente. 
9. Siento que estando dentro del municipio es muy distinta la visión.

10. Estimo que un vehículo es un bien de lujo en esta comuna.

11. Yo creo que pasa (en) en todo orden.

12. Yo sé que no todo es perfecto en la vida.

13. Todos esperan (que) que cambie en algo (...) la situación.

14. Nosotros queremos (que) que el joven (...) (eh) se exprese libremente.

15. (Uno) uno sabe que eso no le (...) no cambia la forma de pensar.

16. Prometen (...) cosas que no van a cumplir.

17. Entiendo que (...) ellos podrían lograr (...) hacer cambios.

18. Yo creo que (es) también es culpa del sistema educacional.

Enunciados de dos períodos emitidos por mujeres puntarenences

1. Creo que sí (...) participaría de las presidenciables (...) pero (ooo) (...) con otro escenario.

2. Toda la gente espera (...) que (eee) (...) la alcaldesa que asuma (...) genere cambios.

3. Pienso que los políticos (...) no encantan a la gente (...) para que confien en sus proyectos.

4. Yo siento que (...) si a un alcalde le va bien o le va mal es porque si nosotros trabajamos o no.

5. Yo creo que no le damos la oportunidad para que realmente piense.

6. Se supone que al final del juego va a llegar una pareja que (...) más (se) (...) se complemente.

7. Asumir (...) que si a un alcalde le va bien o mal es porque uno lo hizo (...) mejor o no tan bien.

Pausas vacías (silencios)

Pausas llenas (alargamientos)

Pausas llenas (vacilaciones)

Total

\section{1 período}

11

3

9

23
2 períodos

13

2

1

16

\section{ANEXO 4}

Zona geográfica de Punta Arenas

Enunciados de un período emitidos por hombres puntarenenses.

1. Percibo que Punta Arenas es más como de (eee) izquierda.

2. No he visto que (eee) los candidatos jóvenes les vaya bien.

3. No creo (que) que haya protagonización de esos jóvenes.

4. Creo que se eligió la persona con más capacidades.

5. Creo que la misma ley les ha ido otorgando muchos (...) (eh) beneficios.

6. Creo que al final (lo) (ooo) la gente joven no se entusiasma (con el) (ell) con el sistema de evaluación. 
7. No vi que haya una vinculación directa ahí.

8. Yo creo que se ven como personas individuales.

9. Yo todavía creo que es una cosa equitativa.

10. Creo que (eee) hay cosas que son de (eee) derechos.

11. Me parece que el pensamiento debe expresarse.

12. Creo que (eee) esa tendencia ha ido cambiando.

13. Creo que es (...) algo sumamente importante para nosotros.

14. Yo creo que el joven magallánico no se vio representado en las votaciones.

15. No creo que así la gente vote.

16. No estoy en acuerdo en que sea tan legal.

17. Aprendí que todo el mundo tiene derecho a (aaa) estar feliz.

18. Él cree que tiene un piso político para (...) presentarse a las elecciones.

Enunciados de dos períodos emitidos por hombres puntarenences

1. Yo creo que lo que le pasa a los jovenes es un poco eso.

2. Yo creo que hay un tema de (eee) (...) que las personas han dejado (de) de verse en un colectivo.

3. Tengo que participar de lo (ooo) (de) de todo ese tipo de actividades (...) que en el fondo son transversales.

4. Yo creo que dado que estas votaciones son $\tan (. .$.$) impares (...) (te) tienen$ más votos.

5. Créanme que va a cambiar porque la gente aquí se va.

6. Yo creo que la instancia de la calle es (...) válida (...) siempre y cuando yo haya votado (ooo) (...) instancias previas de negociación.

7. Se puede decir que Punta Arenas es una región que no está (aaa) identificada con un color ni tendencia política.

Pausas vacías (silencios)

Pausas llenas (alargamientos)

Pausas llenas (vacilaciones)

Total

$\begin{array}{cc}1 \text { período } & 2 \text { períodos } \\ 3 & 7 \\ 8 & 4 \\ 4 & 3 \\ 15 & 14\end{array}$

